\title{
Implantable Bioimpedance Monitor using ZigBee
}

\author{
P. Bogónez-Franco, R. Bragós, member, IEEE, A. Bayés-Genis and J. Rosell-Ferrer, member, IEEE
}

\begin{abstract}
In this paper, a novel implantable bioimpedance monitor using a free ZigBee protocol for the transmission of the measured data is described. The application field is the tissue and organ monitoring through electrical impedance spectroscopy in the $100 \mathrm{~Hz}-200 \mathrm{kHz}$ range. The specific application is the study of the viability and evolution of engineered tissue in cardiac regeneration. Additionally to the telemetric feature, the measured data are stored in a memory for backup purposes and can be downloaded at any time after an RF link break. In the debugging prototype, the system autonomy exceeds 1 month when a 14 frequencies impedance spectrum is acquired every 5 minutes. In the current implementation, the effective range of the RF link is reduced and needs for a range extender placed near the animal. Current work deals with improving this range.
\end{abstract}

\section{INTRODUCTION}

$\mathrm{T}_{\mathrm{n}}$ HE electrical impedance technique can be used to monitor the tissue and organ state. Multifrequency electrical impedance measurements provide information about cell viability, density, morphology and intra-extra cellular fluid ratio, being thus useful to detect macroscopic changes in a tissue [1].

In cardiac tissue characterization applications, electrical impedance spectroscopy (EIS) has been used to detect acute ischemia [2], [3], old infarction scar [4] and rejection in transplanted heart [5], [6]. Measurements are performed in open thorax set-ups [3], through catheter [3], [4] or using a pacemaker-type device [5], [7].

Our group is involved in research projects with the Cardiology Department of Santa Creu i Sant Pau Hospital, in Barcelona, with two running applications: non-invasive graft rejection detection and regenerative tissue engineering. In this last topic, a non-functional myocardium area ( $e g$ an old infarction scar) is intended to regenerate by using myocardial cell precursors derived from stem cells. Our group has developed a technique for in-vitro cell culture monitoring using EIS with interdigitated microelectrodes in a 4-electrode configuration to separate cell-growing and

Manuscript received April 23, 2009. This work was supported in by the MICINN under grant SAF2008-05144-C02-02, by the Generalitat de Catalunya SGR group and by Ministerio de Sanidad through Redes de Investigación del Instituto de Salud Carlos III (project REDINSCOR).

*P. Bogónez-Franco, R. Bragós and J. Rosell are with the Instrumentation and Bioengineering Group (IEB) of the Universitat Politècnica de Catalunya (UPC), c/Jordi Girona 1-3, 08034 Barcelona, Spain. (e-mail: bogonez@eel.upc.edu, rbb@eel.upc.edu, jrosell@eel.upc.edu).

A. Bayés-Genís is with the Cardiology Service of the Hospital de la Santa Creu I Sant Pau, C. Sant Antoni Maria Claret 167, 08025 Barcelona, Spain (e-mail: abayesgenis@santpau.cat) cell-adhesion [8]

In order to monitor the viability and evolution of the engineered tissue after its implantation in an animal model heart (pig or sheep), an implantable device should be developed. The device should be able to acquire an impedance spectrum every 5 minutes in the range $100 \mathrm{~Hz}-$ $200 \mathrm{kHz}$ during 1 month. The design and preliminary results of EIS performance, power consumption and RF range are presented.

\section{METHODS}

\section{A. Bioimpedance monitor}

The bioimpedance monitor developed measures the electrical impedance at 14 frequencies. It stores the data collected and transmits them to an external computer. To allow us to debug the bioimpedance monitor a first prototype has been built over a two-layer PCBwhose dimensions are $60 \mathrm{~mm} \times 60 \mathrm{~mm}$. The final system has a size of $35 \mathrm{~mm} \times 37 \mathrm{~mm}$ and these are mainly given by the battery. It is a six-layer PCB with a height of $8 \mathrm{~mm}, 5 \mathrm{~mm}$ height of the battery and $3 \mathrm{~mm}$ of the PCB with the components.

In figure 1 shows the debug prototype (left) and the final system PCB (right).

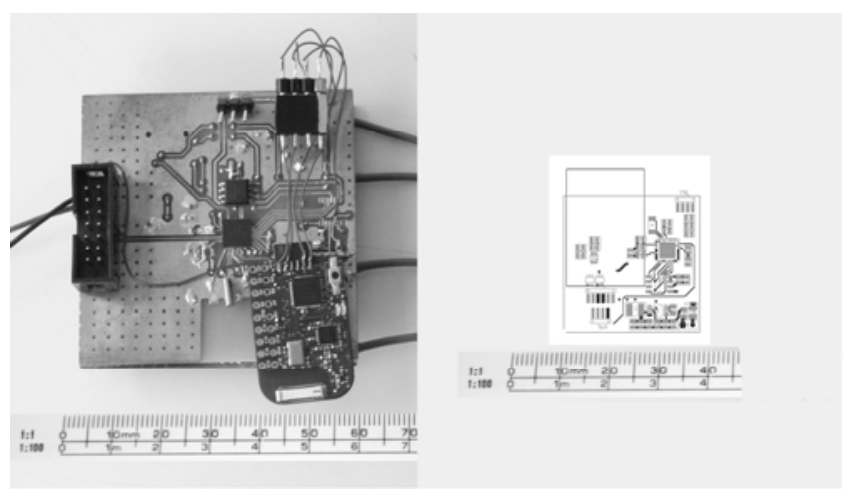

Fig. 1. Picture of the prototype developed for system debugging (left) and the pcb of the final bioimpedance monitor (right).

\section{B. Power supply}

The bioimpedance monitor is powered from a NiMH battery and has two dc-dc converters. The digital circuit of the bioimpedance monitor is powered at 3.3 volts whereas the analog sub-circuits are powered at \pm 2.8 volts. The analog supply can be switched on when the bioimpedance measurements are going to start. Also the supply of the RF transceiver is controlled. When the monitor is in sleep mode 
the only functional system is the microcontroller $32 \mathrm{kHz}$ low-power oscillator.

The system makes a measurement every 5 minutes and remains the most part of time in sleep mode, then increasing the autonomy. Figure 2 shows the current consumption graph for a measurement period of 35 seconds. When the system is in sleep mode the current consumption is $0.2 \mathrm{~mA}$, when it is doing a measurement the current consumption is $35 \mathrm{~mA}$ and extents 2.7 seconds. When it is storing the data in the memory, the consumption is $1 \mathrm{~mA}$ with a extent of $100 \mathrm{~ms}$ and when it is transmitting the data, the current consumption is $11 \mathrm{~mA}$ lasting 2 seconds. The peaks that appear in the figure 2, when the bioimpedance monitor is in sleep mode are due to the microcontroller exits from the sleep mode, when it looks for commands transmitted from the access point.

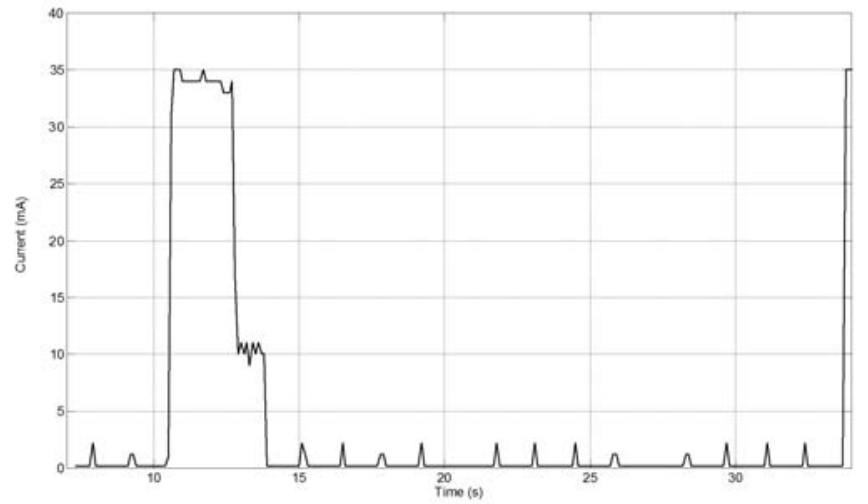

Fig. 2. Current consumption graph during 35 seconds period.

Considering a mean consumption of $0.588 \mathrm{~mA}$ in the 5 minutes period and using a battery with a capacity of 450 $\mathrm{mAh}$, the theoretical autonomy of the system is 765 hours (32 days). Bigger capacity batteries are available.

\section{Bioimpedance measurement system}

The bioimpedance monitor is based in the 12 Bit Impedance Converter and Network Analyzer AD5933 [9], modified with an analog front-end that implements a 4electrode measurement structure and allows to measure small impedances [10]. Basically the main modifications are the addition of a voltage controlled current source, to inject a constant current, a wide-bandwidth differential amplifier, and a voltage to current converter plus a voltage shifter to adapt to the single supply, 2-electrode structure of the device. Figure 3 shows the block diagram of the monitor.

The bioimpedance monitor is tailored to measure in the range $10 \Omega$ to $1 \mathrm{k} \Omega$, at a programmable set of frequencies. The initially chosen frequencies are $100 \mathrm{~Hz}, 200 \mathrm{~Hz}, 500$ $\mathrm{Hz}, 700 \mathrm{~Hz}, 1 \mathrm{kHz}, 2 \mathrm{kHz}, 5 \mathrm{kHz}, 7 \mathrm{kHz}, 10 \mathrm{kHz}, 20 \mathrm{kHz}$, $50 \mathrm{kHz}, 70 \mathrm{kHz}, 100 \mathrm{kHz}$ and $200 \mathrm{kHz}$. The value of the real and the imaginary part measured is the arithmetic mean of 4 measures at each frequency.

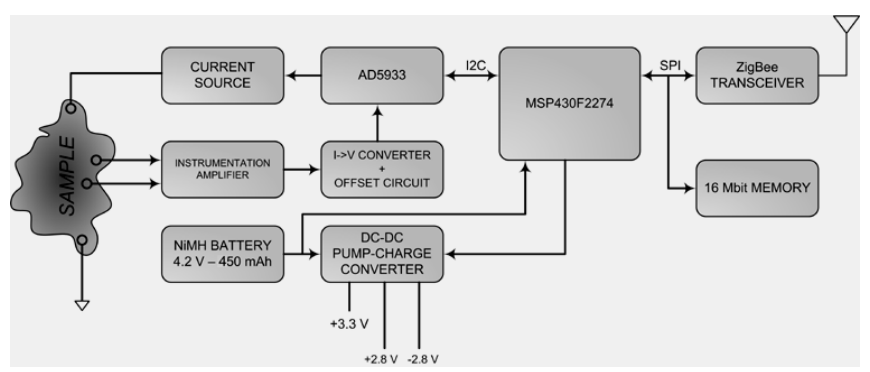

Fig. 3. Block diagram of the bioimpedance monitor.

The current injected to the load is limited to $10 \mu$ App with a bandwidth of $1 \mathrm{MHz}$. Figure 4 shows the variation of the current injected versus load impedance. At $100 \mathrm{~Hz}$ the error in the current injected is $3.2 \%$ and at $200 \mathrm{kHz}$ if $5 \%$ when the load impedance is $980 \mathrm{ohms}$. Part of this error is systematic and can be compensated by calibration.

The connection of the bioimpedance monitor with the electrodes will be done using a $1.8 \mathrm{~mm}$ outer diameter coaxial cables.
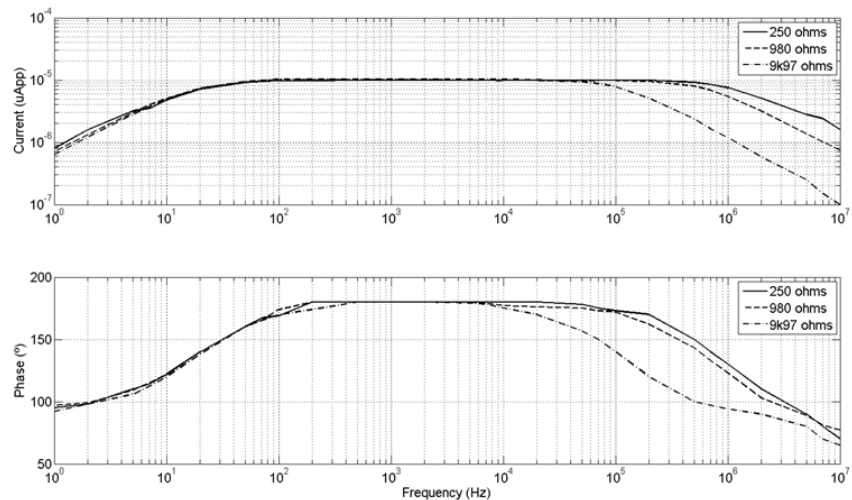

Fig. 4. Magnitude and phase angle of the injected current versus frequency for 3 load impedances $(250 \Omega, 980 \Omega$ and $10 \mathrm{k} \Omega$ ).

\section{Temperature measurement}

For each impedance measurement the temperature is recorded and saved to the system memory. This will give us an extra-information. The temperature measurement is done by the AD5933 and measures the temperature of the pig's body. The increment in temperature due to the power dissipation has small effect on the measure and is $0.1{ }^{\circ} \mathrm{C}$ maximum increment.

\section{E. RF communication}

The data are sent to the PC using a $2.4 \mathrm{GHz} \mathrm{RF}$ transceiver of Texas Instruments running the SimpliciTI protocol. SimpliciTI [11] is a low-power RF network protocol aimed at small RF networks and with a limited number of nodes talking directly to each other, it is a reduced version of the proprietary ZigBee protocol. The topology implemented is a star network, which has an access point (AP), an end device (ED) and a range extender (RE). 
The AP must be always on and its main task is to form the network and act as a coordinator between the nodes. The ED is the bioimpedance monitor and sends the data to the AP. Also, it may accept commands from the AP to modify it behavior. The RE allow to increase the distance between the $\mathrm{AP}$ and the ED. It will be mounted in a harness attached to the skin surface of the animal. The transmitted power is -12 $\mathrm{dBm}$ at a data rate of 9600 Bauds.

\section{F. Memory}

In case the radiofrequency link breaks, each impedance measurement is saved in a memory. The capacity of the memory is 16 Mbits, enough to save the data of 86 days of measurements. The data saved can be downloaded to the PC at any time.

\section{G. PC Software Application}

The data sent by the bioimpedance monitor are received in the computer, using another $2.4 \mathrm{GHz} \mathrm{RF}$ transceiver, saved and plotted in different ways to facilitate the interpretation. Also the application shows the received signal strength indication (RSSI) of the radiofrequency link to check the risk of losing the RF connection.

From this application the user can change the measurement frequencies, perform a single measurement, program the measurement period, erase the memory, and download the data.

\section{H. Packaging}

The implantable bioimpedance monitor will be wrapped with cellophane film $(3 \mathrm{M})$ and sealed with medical-grade silicone (Nusil Med4011). The electrode's cables will be tubed with a silicone tube and cured with epoxy (Devcon).

\section{RESULTS}

\section{A. Electrical impedance measurements}

To study the performance of the measurement system, two $\mathrm{Rp} \| \mathrm{C}+\mathrm{Rs}$ circuits have been used as a measurement load, measuring both resistance and reactance and comparing them with the theoretical model. The RC networks simulate the myocardium impedance.

Figure 5 shows the plot of the imaginary $v s$ real part of one of the $\mathrm{Rp} \| \mathrm{C}+\mathrm{Rs}$ circuits with $\mathrm{Rs}=68 \Omega, \mathrm{Rp}=200 \Omega$ and $\mathrm{C}=82 \mathrm{nF}$.

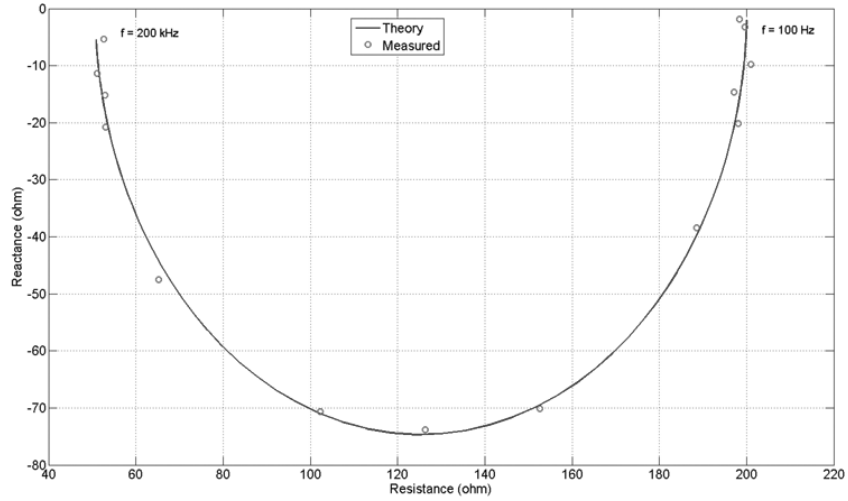

Fig. 5. Impedance plot of a $\mathrm{Rp} \| \mathrm{C}+\mathrm{Rs}$ circuit. $\mathrm{Rs}=68 \Omega, \mathrm{Rp}=200 \Omega$ and $\mathrm{C}=82 \mathrm{nF}$. Frequency range from $100 \mathrm{~Hz}$ to $200 \mathrm{kHz}$.

Figure 6 shows the plot of the second $2 \mathrm{R}-1 \mathrm{C}$ series circuit measured. The values are $\mathrm{Rs}=47 \Omega, \mathrm{Rp}=130 \Omega$ and $\mathrm{C}=$ $100 \mathrm{nF}$.

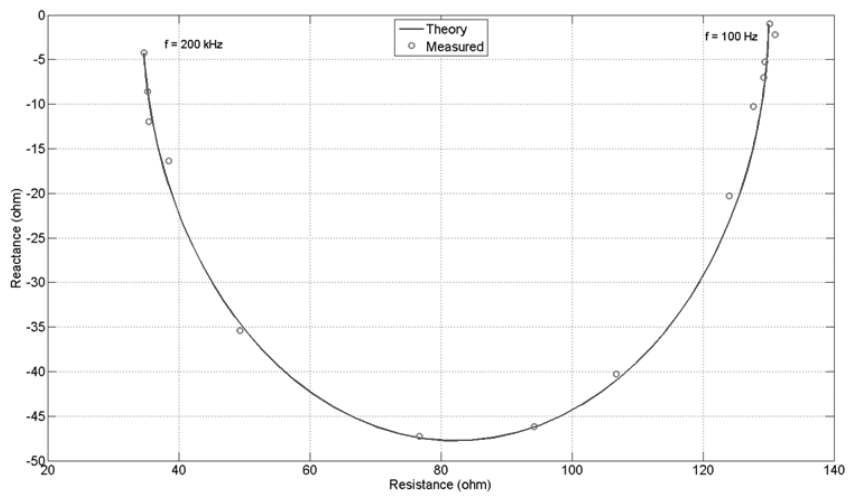

Fig. 6. Impedance plot of a $\mathrm{Rp} \| \mathrm{C}+\mathrm{Rs}$ circuit. $\mathrm{Rs}=47 \Omega, \mathrm{Rp}=130 \Omega$ and $\mathrm{C}=100 \mathrm{nF}$. Frequency range from $100 \mathrm{~Hz}$ to $200 \mathrm{kHz}$.

The error in the estimation of the circuit resistors from the impedance measurements model fitting is below $0.75 \%$.

\section{B. RF range}

Because of the big absorption of the RF signal by the tissues surrounding the implant at $2.4 \mathrm{GHz}$, mainly due to the resonance frequency of the water molecules [12], we perform several measurements to verify the RF range we would expect. The bioimpedance monitor was put into a plastic egg and inside a fishbowl $(30 \mathrm{~cm} \times 15 \mathrm{~cm} \times 15 \mathrm{~cm})$, figure 7. The fishbowl was filled with saline solution with conductivity of $16 \mathrm{mS} / \mathrm{cm}$, similar that most tissues inside the body until the level reached $13 \mathrm{~cm}$. 


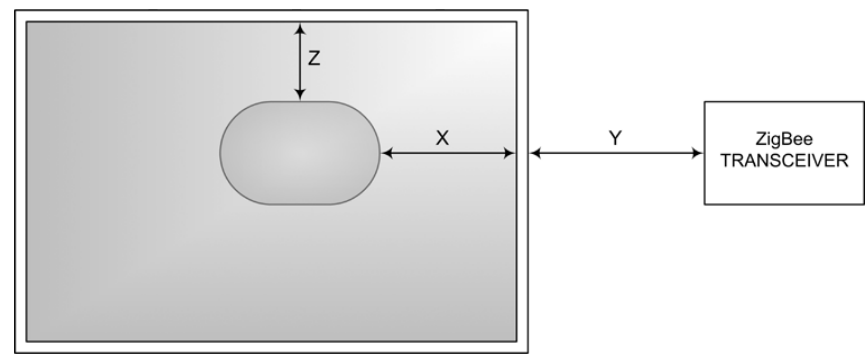

Fig. 7. Bioimpedance monitor inside the fishbowl to measure the RF range of the link.

The measurement consists in moving the plastic egg in the longitudinal direction (x) and in the depth direction (z) and measure the distance (y) at which the RF link gave communication errors. Figure 8 shows in a plot the RF link break distance as function of the longitudinal and depth direction of the plastic egg inside the fishbowl. The distance $\mathrm{y}$ exceeds $1 \mathrm{~m}$ when the device is close to the tank perimeter $(\mathrm{x}<1 \mathrm{~cm})$ and reduces to $70 \mathrm{~cm}$ when it is $6 \mathrm{~cm}$ into the tank.

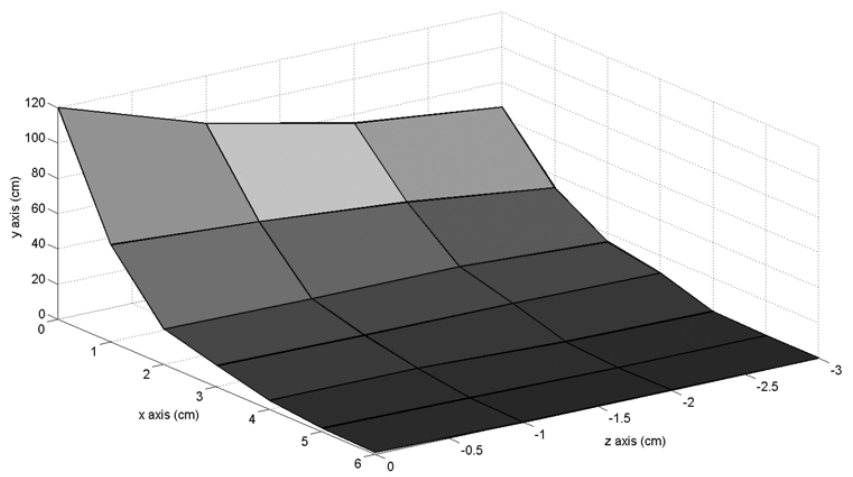

Fig. 8. RF range of the link with the bioimpedance monitor immersed in saline solution.

\section{DISCUSSION AND CONCLUSIONS}

We measured a low RF range when the bioimpedance monitor is immersed in a few centimeters of saline solution. The range we can expect in the clinical application may be greater because normally the implanted devices are not deeply inside the body but are placed transcutaneous [13].

With the preliminary results reported in this work it would be possible to implant the bioimpedance monitor to study the evolution of engineered tissue and transmit the data wirelessly using SimpliciTI, the free ZigBee protocol. A range extender should be placed in a harness in the animal surface. In order to raise the RF range without employing a $\mathrm{RE}$, we will try to increase the sensitivity of the receiver, using a directional antenna with a low-noise amplifier in the receiver part. This point is currently investigated and will be reported.

The results show the viability of real-time impedance spectroscopy measurements for organ or tissue monitoring using a telemetric device.

\section{REFERENCES}

[1] E. Gersing, "Impedance spectroscopy on living tissue for determination of the state of organs," Bioelectrochem. Bioenergetics, vol. 45, no. 2, pp. 145-149, May 1998.

[2] M.M. Gebhard, E. Gersing, C.J. Brockhoff, P.A. Schnabel, H.J. Bretschneider. "Impedance spectroscopy: a method for surveillance of ischemia tolerance of the heart". Thorac. Cardiovasc. Surg. 1987, vol 35, no. 1, Feb 1987, pp. 26-32.

[3] Y. Salazar, R. Bragos, O.Casas, J. Cinca and J. Rosell, "Transmural versus nontransmural in situ electrical impedance spectrum for healthy, ischemic, and healed myocardium," IEEE Trans. Biomed. Eng., vol. 51, no. 8, Aug. 2004, pp. 1421-1427.

[4] M. Warren, R. Bragós, O. Casas, A. Rodriguez-Sinovas, J. Rosell, I. Anivarro and J. Cinca, "Percutaneous electrocatheter technique for online detection of healed transmural myocardial infarction," Pacing Clin. Electrophysiol., Aug. 2000, vol 23, no. 8, pp. 1283-1287.

[5] R. Pfitzmann, J. Müller, O. Granhan and R. Hetzer, "Intramyocardial impedance measurements for diagnosis of acute cardiac allograft rejection," Ann. Thorac. Surg., Aug. 2000, vol. 70, no. 2, pp. 527-532.

[6] J. Cinca, J. Ramos, M.A. Garcia, R. Bragós, A. Bayés-Genís, Y Salazar, R. Bordes, S. Mirabet, J.M. Padró, J.G. Picart, X. Viñolas, J. Rosell-Ferrer, "Changes in myocardial electrical impedance in human heart graft rejection," Eur. J. Heart Fail., vol. 10, no. 6, Jun. 2008, pp. 594-600.

[7] A. Kink A, R.W. Salo, M. Min, T. Parve T, I. Rätsep. "Intracardiac electrical bioimpedance as a basis for controlling of pacing rate limits”. Conf .Proc. IEEE Eng. Med.Biol. Soc. 2006. vol. 1, pp. 63086311.

[8] R. Bragós, E. Sarro, A. Fontova, A. Soley, J. Cairó, A. Bayés-Genís and J. Rosell, "Four versus two-electrode measurement strategies for cell growing and differentiation monitoring using electrical impedance spectroscopy," Conf. Proc. IEEE Eng. Med. Biol Soc., Aug. 2006, vol. 1, pp. 2106-2109.

[9] (2008), “AD5933”, [Online]. Available: www.analog.com.

[10] F. Seoane, J. Ferreira, J.J. Sánchez and R. Bragós, "An analog frontend enables electrical impedance spectroscopy system on-chip for biomedical applications," Physiol Meas, vol. 6, 29, pp. S267-78, June 2008.

[11] (2009), "SimpliciTI", [Online]. Available: www.ti.com.

[12] P. Valdastri, A. Menciassi and P. Dario, "Transmission power requirements for novel ZigBee implants in the gastrointestinal tract," IEEE Trans Biomed. Eng., Jun. 2008, vol. 55, no. 6, pp. 1705-1710.

[13] K. Ellenbogen, "Cardiac pacing," Blackwell Scientific, London, 1992. 